\title{
Penser la décroissance avec Gilbert Simondon
}

\author{
Jamil Alioui \\ Faculté des lettres, Section de philosophie, Université de Lausanne, Lausanne, Suisse \\ Auteur-ressource. Courriel : jamil.alioui@alambicbicephale.com
}

\begin{abstract}
Résumé
Dans cet article, nous étudions l'axiologie des techniques des discours écologistes de la décroissance à l'aune de celle du philosophe français Gilbert Simondon. Cette articulation fertile permet de montrer, premièrement, que les discours de la décroissance n'évaluent les techniques qu'à partir de leurs usages; deuxièmement, elle explique pourquoi une telle axiologie, praxéologique plutôt que technologique, est incapable d'influer sur le progrès technique. À partir de Simondon, nous montrons notamment que la pensée de la décroissance ignore la distinction entre information et énergie au sein des réalités techniques, distinction pourtant nécessaire si l'on souhaite relier l'étude des techniques et l'écologie de façon adéquate et constructive.
\end{abstract}

\begin{abstract}
In this article, I examine the axiology of techniques in the environmentalist discourses of degrowth through the lens of French philosopher Gilbert Simondon. This fertile articulation shows, first, that degrowth discourses evaluate techniques only based on their uses; second, it explains why such an axiology, praxeological rather than technological, is unable to influence technical progress. Beginning with Simondon, I show in particular that degrowth thinking ignores the distinction between information and energy within technical realities. Yet, this distinction is necessary if we wish to link the study of techniques and ecology in a constructive way.
\end{abstract}

Mots-clés : décroissance ; écologie ; technologie ; Simondon ; information ; progrès ; techniques

Le mouvement écologique est très précieux parce qu'il contient une éthique constructive qui a ses normes et peut-être aussi ses limites comme toute normativité.

Simondon (2014, p. 344)

La « décroissance » est le mot d'ordre d'un ensemble de discours et de mouvements écologistes qui luttent contre le productivisme, c'est-à-dire contre la présupposition plus ou moins consciente de la possibilité d'une croissance économique infinie, et contre les conséquences de cette présupposition. Ainsi, ceux qui se font appeler les

(C) The Author(s), 2021. Published by Cambridge University Press on behalf of the Canadian Philosophical Association/ Publié par Cambridge University Press au nom de l'Association canadienne de philosophie. This is an Open Access article, distributed under the terms of the Creative Commons Attribution licence (https://creativecommons.org/licenses/by/4.0/), which permits unrestricted re-use, distribution, and reproduction in any medium, provided the original work is properly cited. 
« objecteurs de croissance ${ }^{1}$ ne se contentent pas d'accuser les grands pollueurs d'exploiter de façon irresponsable les ressources naturelles en menaçant la biodiversité et en ruinant les écosystèmes ; ils attaquent aussi par ailleurs les partisans de la " croissance verte » ou du « développement durable », qu'ils jugent hypocrites. Selon les discours de la décroissance, en effet, que la croissance soit " néolibérale ", " écologique " ou « socialiste » est indifférent, car les problèmes qu'il s'agit de résoudre - c'est leur hypothèse - ont pour cause le productivisme lui-même, comme idéologie de la croissance, ainsi que la relation problématique aux énergies et aux matières nonrenouvelables que sa mise en œuvre implique nécessairement. Ainsi, "la crise écologique et sociale actuelle ne peut pas trouver sa solution dans un surcroît de croissance économique » (Flipo, s.d.). De façon plus théorique, le productivisme est aussi entendu par le courant de la décroissance comme un Grand Récit ${ }^{2}$, celui de l'inéluctabilité et de la continuité quasi naturelle du progrès des sciences et des techniques. La critique considère que le productivisme en tant qu'idéologie est un mythe dominant et majoritaire qui a pour corrélat malheureux la mise en péril de la possibilité de discuter et de négocier politiquement et démocratiquement les finalités des sciences et des techniques.

Nous nous intéressons ici en particulier à l'axiologie des techniques propre aux discours de la décroissance, axiologie que nous aimerions saisir et problématiser en l'articulant à celle que propose le philosophe Gilbert Simondon. Nous montrons d'abord que les discours de la décroissance n'évaluent les techniques qu'à partir de leurs effets et de leurs usages. Nous expliquons ensuite, avec Simondon, en quoi une telle évaluation manque la relation entre les fonctionnements et les structures, essentielle aux réalités techniques, et en quoi elle est insuffisante si, comme les objecteurs de croissance, l'on cherche à avoir une influence sur le progrès technique. Nous indiquons alors ce que peut être une évaluation proprement technologique des techniques, puis, à partir de la distinction simondonienne entre information et énergie, nous précisons finalement comment une telle évaluation contribue à l'instauration d'une "culture technique ", dans laquelle humains et techniques entrent en relation $\mathrm{d}^{\prime}$ " invention perpétuée ». Nous concluons en montrant comment l'axiologie des techniques de Simondon dialogue avec la pensée de la décroissance, dans l'horizon de problèmes communs aux deux perspectives.

\section{Le problème de l'évaluation non-technologique des techniques dans les discours de la décroissance}

L'économie, écrivent les objecteurs de croissance, [...] a baptisé " externalité négative " l'ensemble des effets occasionnés par les échanges économiques

\footnotetext{
${ }^{1}$ L'expression « objecteurs de croissance », employée par les tenants des discours de la décroissance eux-mêmes, semble apparaître pour la première fois en 1973, dans le titre d'un numéro de la revue catholique Les cahiers de la Nef, "Les Objecteurs de croissance : prospérité, oui... mais à quel prix ? ", $30^{\text {ème }}$ année, J. Tallandier éditeur.

${ }^{2}$ On trouvera une telle approche avant tout chez Serge Latouche, qui parle d'" économicisme » (2003, p. 26) et qui évoque les «bases imaginaires » de la société de consommation (2019, p. 13). En particulier, on lui doit l'introduction de l'expression " décolonisation de l'imaginaire " au sein de la pensée de la décroissance, employée d'ailleurs comme titre d'un de ses ouvrages et reprise notamment par Bernard Guibert (2004) et Michel Lepesant (2015) sous la forme d'une critique de l'« imaginaire de la croissance».
} 
privés et dont le coût est reporté sur la collectivité. Cette définition purement analytique ne conduit à aucune solution claire pour ce qui est de la manière de réduire ces externalités. Elle ne conduit pas non plus à un accord politique sur leur étendue et leur gravité. Son effet le plus sûr est de retirer le problème du débat public pour le confier à des experts, sans que soit remis en cause le concept habituel de productivité et donc de progrès technique. [...] Pour les objecteurs de croissance, le problème n'est pas d' " internaliser les externalités » de manière technocratique, mais de mettre en cause la rationalité économique au profit d'une autre rationalité s'appuyant sur une autre conception de la nature, de la liberté et des droits (Bayon et al., 2012, ch. 4).

Ce passage, issu d'un récent ouvrage de référence, formule l'un des constats inauguraux de la pensée de la décroissance : le productivisme de certains a des conséquences délétères pour tous. Dans la mesure où l'objectif d'une croissance perpétuelle de la production engendre des effets écologiques, économiques et sociologiques négatifs, les objecteurs de croissance reprochent aux tenants du productivisme de refuser de remettre en discussion cet objectif. Les productivistes sont conscients de ces effets globaux néfastes, mais, selon la critique, leur réponse se limite toutefois au financement de "solutions sparadrap " localisées et choisies par des " experts ", c'est-à-dire de façon non démocratique. Selon la critique toujours, le problème est donc plus profond et plus urgent que ce que veulent bien admettre les productivistes tenus pour responsables ; il requiert davantage qu'une simple budgétisation des coûts liés aux effets négatifs : il requiert une sortie de l'idéologie productiviste et l'instauration d'une nouvelle façon de penser.

Ce qui nous intéresse particulièrement ici est la relation immédiate que les auteurs établissent entre le " concept habituel de productivité » et le "progrès technique ». Le fait que la méthode ad hoc qu'est l' " internalisation des externalités » soit dite " technocratique " est aussi remarquable. En tant qu'il concerne l'accès à des techniques ainsi que leur emploi - il s'agit en l'occurrence de techniques comptables autant que de production - le terme " technocratique " doit être mis en relation avec la question générale de l'usage des techniques ; il s'oppose à " démocratique ». De plus, en rapprochant de la sorte le "concept habituel de productivité » et le " progrès technique ", la critique du productivisme rapporte les évolutions et le développement des techniques tant aux moyens d'accroître la production qu'à ceux de produire les sparadraps à appliquer sur les " dommages collatéraux » causés par cette production. En ce sens, la critique du productivisme formulée dans les discours de la décroissance semble considérer l'ensemble des techniques comme quelque chose de négatif. Elle rapporte, du moins, leurs progrès à ceux des problèmes qu'il s'agit de résoudre.

Toutefois, contrairement à ce que peut suggérer la lecture du texte précédent, toutes les techniques ne sont pas à condamner pour les objecteurs de croissance; il existe des types de réalités techniques ne faisant pas référence au " concept habituel de productivité "; certaines techniques sont compatibles avec le projet politique et socio-économique de la décroissance. Serge Latouche, par exemple, évoque à juste titre la possibilité d'une "écoconception des produits" (Latouche, 2019, pp. 54 et 57). Philippe Bihouix, connu notamment pour son travail sur la distinction entre 
« high tech » et « low tech » (Bihouix 2014), rappelle la possibilité et l'intérêt commun de techniques réparables ou durables. De telles exigences s'inscrivent dans le sillage de la valorisation de l'agriculture organique (à laquelle correspond une réprobation argumentée de l'agriculture mécanique) soutenue par Nicholas Georgescu-Roegen dans sa théorie économique de la décroissance ${ }^{3}$. Dans les éléments de bioéconomie de cette même théorie (NGR, p. 147-149), le statisticien roumain suggère de limiter la production de « gadgets extravagants » et les effets de consommation liés à la mode. Denis Bayon, Fabrice Flipo et François Schneider évoquent quant à eux les techniques "non-industrielles » et "non-modernes ", telles que l'usage du margousier comme antibactérien naturel ou celui de totems pour protéger les espèces biologiques nécessaires à la stabilité de l'écosystème (Bayon et al., 2012, ch. 1). Ces axiologies des techniques, autrement dit ces exigences qui permettent de juger et donc de distinguer le «bon » du " mauvais » progrès technique, ont presque toutes pour racine le concept de « convivialité » d'Ivan Illich, auquel la plupart des objecteurs de croissance se réfèrent. Selon Illich, les techniques peuvent être dites " conviviales » et désirables quand elles sont " au service de la personne intégrée à la collectivité, et non au service d'un corps de spécialistes », quand elles " ne [suscitent] ni esclaves ni maitres » et quand « l'usage que chacun en fait n'empiète pas sur la liberté d'autrui d'en faire autant " (Illich, 1973, pp. 13, 27 et 45).

Or, il convient de remarquer que la pensée de la décroissance distingue le «bon » progrès technique du " mauvais » à partir de critères axiologiques qui, en fait, n'évaluent pas la technicité elle-même. En l'occurrence, ces critères n'évaluent des techniques que leurs effets et leurs usages; ils attribuent une valeur aux réalités et progrès techniques, désirables ou indésirables, non pas à partir des fonctionnements, des opérations ou encore des structures, mais en fonction de certaines façons de les utiliser. En effet, lorsque les objecteurs de croissance évaluent par exemple la technique de l'aviation à partir de son " coût social réel ", critiqué à juste titre car " caché, "externalisé", reporté dans l'avenir ou sur les parties les plus faibles de la population » (Bayon et al., 2012, ch. 1), leur critique ne concerne ni des opérations ni des structures stricto sensu techniques - telles les propriétés de certains matériaux, la forme du fuselage, le fonctionnement du moteur ou la conception des organes de commande - qui seraient spécifiques au productivisme ; elle ne vise que les effets de ces réalités techniques, lorsqu'elles sont utilisées par l'idéologie productiviste. De surcroit, la distinction entre une technique " conviviale " et une technique "nonconviviale " ne porte pas sur les opérations, les structures ou les schèmes techniques eux-mêmes ; elle ne concerne que les usages de ces opérations et structures et leurs effets relativement à ces usages. En particulier, Illich définit les techniques conviviales comme des techniques que " chacun peut utiliser, sans difficulté [...] à des fins qu'il détermine lui-même " (Illich, 1973, p. 45). En un mot, ce que la décroissance voudrait rapporter à la technique n'est d'abord qu'un ensemble d'usages possibles des techniques par les humains ; pour cette raison, nous affirmons que la pensée de la

\footnotetext{
${ }^{3}$ « Le remplacement du buffle par le tracteur, du fourrage par les combustibles pour les moteurs, du fumier et de la jachère par des fertilisants chimiques équivaut à substituer des éléments rares au plus abondant de tous, le rayonnement solaire " (Georgescu-Roegen, La décroissance. Entropie. Écologie. Économie, 2008, p. 138 ; dorénavant abrévié NGR dans le corps du texte).
} 
décroissance fait recours à une évaluation non-technologique des réalités techniques. Voyons maintenant en détail, avec Simondon, pourquoi exactement une évaluation de la technique par ses usages et par les effets de ces usages est problématique.

\section{Le sens et la valeur d'une évaluation technologique des techniques}

Commençons par montrer comment Simondon distingue les usages des techniques à partir de la problématique de la relation entre fonctionnements opératoires dynamiques et structures physiques et matérielles stables.

L'usage, écrit Simondon, réunit des structures et des fonctionnements hétérogènes sous des genres et des espèces qui tirent leur signification du rapport entre ce fonctionnement et un autre fonctionnement, celui de l'être humain dans l'action ${ }^{4}$.

L'usage, tel que le définit Simondon, ne concerne que la relation entre, d'un côté, une réalité technique - opération ou structure ici " hétérogènes ", c'est-à-dire sans relation - et, de l'autre, l'action de l'être humain. D'un point de vue épistémologique, l'étude des usages est donc une praxéologie plutôt qu'une technologie; son objet de référence, celui à partir duquel, par exemple, une typologie ou une axiologie deviennent possibles, est l'être humain et/ou ses actions ; du moins, cet objet n'a rien d'essentiellement technique. On trouvera un très bon exemple d'une telle approche dans l'étude de la bicyclette que propose Illich en 1975 dans Énergie et équité :

Le roulement à billes a été inventé il y a un siècle. [...] Il a rendu possible l'invention de la bicyclette. [...] À bicyclette, l'homme va de trois à quatre fois plus vite qu'à pied, tout en dépensant cinq fois moins d'énergie (Illich, 2003, p. 105, cité dans Paquot, 2012).

Dans ce passage, la condition de possibilité des réalités techniques nommées « roulement à billes » et "bicyclette » est ignorée ; en l'occurrence, l'intérêt et la valeur portés à ces réalités ne sont motivés que par la question de l'économie humaine d'énergie dans l'action. Une approche praxéologique peut présenter des intérêts certains ; elle devient problématique, cependant, lorsqu'elle prétend vouloir connaître, classer ou évaluer les techniques, car elle laisse dans l'ombre le problème, pourtant central et décisif, de "la convergence des fonctions dans une unité structurale " (MEOT, p. 26) qui, selon Simondon, est le problème proprement technique. Pour cette raison, il est dit de l'usage qu'il est " une spécificité illusoire » (MEOT, p. 21) de l'objet technique, qu'il en est une propriété fictive, inessentielle (MEOT, p. 334), uniquement apparente et donc incapable d'en soutenir une typologie ou une évaluation. C’est que la correspondance entre les structures constituant les réalités techniques et les problèmes pratiques propres à l'« humain dans l'action » n'est jamais donnée d'emblée ; Simondon écrit en effet : « aucune structure fixe ne correspond à

\footnotetext{
${ }^{4}$ Simondon (Du mode d'existence des objets techniques, 2012, p. 22 ; dorénavant abrévié MEOT dans le corps du texte).
} 
un usage défini » (MEOT, p. 21). Autrement dit, la possibilité qu'une réalité telle que le roulement à billes ou la bicyclette existe dans le monde est a priori sans relation avec le problème humain d'une mobilité énergétiquement plus économique. Une réflexion qui prétend porter sur les techniques ne peut donc pas se contenter d'aborder son objet à partir des usages ; elle doit plutôt connaître, classer et évaluer les manières dont les fonctionnements et les structures convergent dans des unités objectives et organiques.

Insistons encore, avant d'aborder cette convergence, sur cette distinction entre qualité d'usage et qualité technique d'un objet, et arrêtons-nous pour cela sur l'exemple concret, proposé par Simondon, de l'herminette détériorée. "Pratiquement, une herminette peut être en mauvais état, mal affûtée, sans être pourtant un mauvais outil » (MEOT, p. 88). Cet exemple rend attentif au fait qu'un objet inutilisable, dépourvu d'usage possible, n'est pas nécessairement moins technique qu'un objet utilisable. De là, Simondon met en relation d'un côté ce qu'il nomme "éléments techniques » et de l'autre ce qu'il nomme " ensembles techniques ", en montrant que la simple mise en forme d'un bloc de métal homogène ne suffit pas à la fabrication d'une herminette digne de ce nom. La fabrication d'un tel outil, en l'occurrence, requiert l'ensemble technique que forment la fonderie, la forge et la trempe. Les fonctionnements de cet ensemble, qui se caractérise par sa complexité, sa systématicité et son déploiement étendu, permettent donc à l'herminette, objet détachable, de taille humaine, d'actualiser certaines qualités proprement et irréductiblement techniques les éléments techniques —, parmi lesquelles le pouvoir de " recevoir et conserver un bon affûtage même lorsqu'on l'emploie pour travailler dans les bois durs » (MEOT, p. 88). Ce sont bien ces qualités-là, et non les usages, qui permettent d'attribuer leur technicité aux objets techniques.

Par ailleurs, si les techniques doivent être évaluées en tant que telles, c'est aussi pour des raisons politiques. Toute technique doit déjà avoir été inventée et doit déjà avoir fonctionné pour pouvoir être utilisée. Par conséquent, la volonté de légiférer sur les progrès techniques à partir d'une évaluation des usages ne peut travailler que trop tard. Si la fin du technocratisme productiviste et l'instauration de la convivialité impliquent les techniques, alors les objecteurs de croissance doivent avoir la capacité d'influer activement sur ces dernières au moment de leurs genèses. Or, ici, l'abord non-technologique des réalités techniques empêche l'accès aux techniques antérieurement à l'existence des objets fonctionnels, utilisables, déjà inventés, concrétisés et donc potentiellement nuisibles; il entrave la connaissance des « conditions dans lesquelles l'objet technique n'abîme pas la nature » (Simondon, 2014, p. 452). En montrant que l'abord des techniques en tant que telles doit nécessairement porter sur les genèses - autrement dit : en montrant que la technologie doit être génétique - Simondon explique l'impasse politique inhérente aux abords non-technologiques des techniques. L'objet propre de la technologie réside dans un ensemble de convergences, existant en nombre fini, entre des structures et des opérations. Ce sont bien ces convergences, ces façons de devenir " organique » (MEOT, p. 69 ; Simondon, 2015, p. 82) propres aux objets techniques qui, en tant que processus non-arbitraires, tracent les lignes du progrès stricto sensu technique (MEOT, p. 65). 
Il paraît adéquat, dans le cadre d'une étude technologique et génétique des techniques, de commencer par s'interroger sur ce qu'est exactement l'invention, ainsi que sur ce qui la rend possible. Dans cet horizon, l'étude de l'invention que propose Simondon présente des résultats particulièrement intéressants pour l'écologie ${ }^{5}$, en ceci qu'elle n'accorde le titre d'" invention " qu'aux réalités techniques individuelles saisies ou pénétrées avec et dans le milieu au sein duquel elles doivent fonctionner. L'invention, en effet, est un processus selon lequel

les éléments qui matériellement constitueront l'objet technique, et qui sont séparés les uns des autres, sans milieu associé avant la constitution de l'objet technique, doivent être organisés les uns par rapport aux autres en fonction de la causalité circulaire qui existera lorsque l'objet sera constitué (MEOT, p. 71).

L'invention présuppose donc une certaine intuition de l'unité formée par le monde et l'objet technique à venir; elle est conditionnée par ce que Simondon nomme une "virtualité " ${ }^{6}$, et ne devrait pas être confondue avec la simple représentation ou figuration du monde dans ou pour le sujet. "Inventer, c'est faire fonctionner sa pensée comme pourra fonctionner une machine " (MEOT, p. 192), ce qui signifie que la pensée, de façon analogue à la machine, doit concorder opérativement, coïncider fonctionnellement, s'ajuster dynamiquement avec la nature du milieu de la machine à venir, selon des lois qui ne peuvent ni être exclusivement celles de la pensée (comme le sont par exemple les formes a priori de la sensibilité), ni être objectivement données "par la nature ". Pour cette raison, une représentation ou une figuration statique ou théorique de la nature ne suffisent pas à rendre possible l'invention ; cette dernière requiert un isodynamisme, une analogie d'opérations entre la pensée et le monde.

Si le dynamisme de la pensée de l'inventeur donne lieu à un objet, alors le fonctionnement de cet objet signifie une certaine intelligence du milieu. Autrement dit, une invention technique digne de ce nom doit être capable, en tant que telle, de fonctionner avec et selon son milieu, ce qui implique réciproquement un certain " jeu ", une certaine marge d'indétermination de la machine relativement à ce milieu opératoire qui est le sien. C'est d'ailleurs la nécessité de cette marge d'indétermination qui explique, chez Simondon, pourquoi l'" automatisme est un assez bas degré de perfection technique » et pourquoi se persuader du contraire est une «faute logique " (MEOT, p. 12). Comme l'explique Jacques Roux, «l'objet technique inventé se distingue [...] en ce qu'il nécessite dans son fonctionnement

\footnotetext{
${ }^{5}$ Selon Simondon, l'écologie est un mouvement « très précieux » qui « fonde un certain type de prospective du devenir de l'homme dans son milieu ", capable tant de libérer une " charge d'irrationnel "lorsqu'elle conduit par exemple à un "monachisme de type anachorétique " - que de contribuer constructivement à « la prise de conscience de la nécessité de ne pas miser uniquement sur les énergies dures et de savoir s'équiper pour tirer parti des énergies renouvelables". Sur la conception simondonienne de l'écologie, voir "Trois perspectives pour une réflexion sur l'éthique et la technique " (Simondon, 2014, p. 337 et suivantes).

${ }^{6}$ Bernard Aspe reformule la définition simondonienne du virtuel d'une façon particulièrement éclairante : "Le "virtuel" n'est pas ce qui est préformé ou préfiguré, mais ce par quoi vient à exister ce qui n’a d'être que d'avoir été anticipé ; il est le potentiel envisagé selon le temps " (Aspe, 2013, p. 153).
} 
l'intervention pratique de son milieu associé "(Roux, 2002, p. 130). Or, ceci a une conséquence remarquable : une invention dont le fonctionnement détruit le milieu peut être considérée comme techniquement mauvaise sans même requérir d'évaluation économique ou sociologique. En effet, un objet technique qui détruit le milieu pour lequel il a été inventé, in fine, ne peut que se détruire lui-même ; or, la condition minimale d'existence d'un objet technique, qui est aussi l'une des formes les plus élémentaires de la technicité, est la solidité (Simondon, 2005, p. 170). On voit ainsi l'étude des techniques et l'axiologie converger dans ce qui pourrait alors véritablement opérer comme le critère d'une politique des techniques digne de ce nom, pour ne pas dire d'une "écologie des techniques» (Petit, 2016, p. 128).

Lorsque le processus d'invention aboutit, l'existence de l'objet technique peut alors être entendue comme la manifestation d'un succès, en l'occurrence celui de la pensée d'un vivant qui, accédant à l'intuition adéquate du monde - monde qui est aussi celui dans lequel il vit - , découvre une nouvelle virtualité. " Le dynamisme de la pensée, lors de l'invention, s'est converti en formes fonctionnantes » (MEOT, p. 192). L'existence de l'objet, qui aurait pu être empêchée par une intuition inadéquate, atteste alors d'une valeur irréductiblement technique : en effet, si l'opération technique «met en jeu les lois véritables de la réalité naturelle » (MEOT, p. 346), alors la présence de l'objet informe sur la coïncidence des lois du monde naturel et de la pensée humaine qui l'a inventé. Par là, l'invention n'est ni simple transformation de matière ni représentation matérialisée ni actualisation d'un préexistant ; en ce sens elle est irréductible tant à la production qu'à la pensée ou encore à la découverte (Chateau, 1994, p. 125-126).

La communauté opératoire entre la machine et son milieu est donc analogue à la communauté opératoire entre l'inventeur et la nature telle que la symbolise la machine qui fonctionne. En ce sens, les réalités techniques mettent en relation l'humain et le monde. L'opération, comme condition de possibilité de l'invention et de l'existence de l'objet technique, «n'est [donc] pas arbitraire, ployée en tous sens au gré du sujet selon le hasard de l'utilité immédiate" (MEOT, p. 346). Certains lecteurs de Simondon conçoivent ce conditionnement comme un schématisme, à l'instar de Jean-Claude Beaune, qui repère le conditionnement d'un "schématisme originaire " (Beaune, 1990, p. 59), ou de Vincent Beaubois, qui évoque le rôle d'un "schématisme pratique de l'imagination » (Beaubois, 2015). Nous indiquons simplement que l'invention ratifie la réalité d'une analogie d'opérations - Simondon parle aussi de " compatibilité » - entre le monde physique et le monde intellectuel. En ce sens, l'existence de l'objet technique peut effectivement devenir le symbole d'une relation entre l'humain et le monde.

Toutefois, l'invention n'est que le « début d'une lignée d'objets techniques », et cette lignée ne commence qu'avec un « objet abstrait » qui est

la traduction en matière d'un ensemble de notions et de principes scientifiques séparés les uns des autres en profondeur, et rattachés seulement par leurs conséquences qui sont convergentes pour la production d'un effet recherché (MEOT, p. 56).

Postérieurement, l'objet concret est marqué par la " réduction des incompatibilités » et la " redistribution des fonctions en sous-ensembles synergiques », auxquelles préside 
une " essence technique », issue de l'invention inaugurale (MEOT, p. 53). L'étude du développement du Diesel dans le contexte des moteurs à combustion, celle de la transformation de la diode en penthode dans le contexte des tubes électroniques, ou encore celle des différentes étapes qui, via différentes roues à moyeux, mènent de la série de rondins à la perfection du roulement à bille (Simondon, 2005, p. 96), sont autant d'exemples d'études génétiques de la concrétisation que l'on trouve dans le texte. Selon Simondon, c'est très exactement ce processus de concrétisation technique qui caractérise le progrès technique essentiel. Ce dernier peut alors être compris comme une tendance de l'objet technique à devenir organique, c'est-à-dire une tendance à devenir l'analogue d'un système « [laissant] moins de liberté au constructeur » et « moins de tolérance à l'utilisateur " (MEOT, p. 53). L'objet qui se concrétise se détermine; il se sature et s'individualise, comme les objets que l'on trouve dans la nature. En ce sens, l'objet technique abstrait est encore très artificiel, dans la mesure où l'on entend par " artificialité " la " dépendance à l'égard de l'homme due à la séparation entre les fonctions » (Barthélémy, 2005, p. 183) ; réciproquement, on peut dire de l'objet concret qu'il s'est naturalisé.

Mais alors le discours de Simondon interpelle ici l'objecteur de croissance : le fait de concevoir la concrétisation technique des objets comme un processus de naturalisation pose la délicate question de la possibilité d'une emprise de l'humain sur ce progrès. Il semble bien que l'exercice d'une relation entre humain et progrès technique ne puisse ni revêtir la forme d'une action directe, ni faire l'objet, à proprement parler, d'une décision ${ }^{7}$. Dans ce cas, en quoi la proposition de Simondon diffère-t-elle du Grand Récit soutenu par le productivisme et critiqué par les objecteurs de croissance ? La réponse doit être trouvée, nous allons le voir, à partir de la notion de culture technique.

\section{Information, énergie et culture technique}

Les objets techniques naturalisés ont ceci de particulier qu'ils peuvent, aussi légitimement que les organismes naturels, être étudiés de façon inductive. Simondon, dans le sillage de Jacques Lafitte, intitule une telle étude " technologie générale ou mécanologie » (MEOT, p. 58). Étudier technologiquement les objets techniques, c'est les étudier génétiquement en tant que traces ${ }^{8}$ d'une compatibilité qui «a été découverte empiriquement » entre la pensée et le monde ${ }^{9}$. Or, cette technologie générale permet, écrit Simondon, d'" assurer l'insertion des techniques dans le monde d'une manière qui dépasse l'empirisme " (MEOT, p. 299). Autrement dit,

\footnotetext{
${ }^{7}$ Le commerce et l'économie, cherchant à " présenter un objet récent comme supérieur aux plus anciens ", exigent de l'objet un "faux renouvellement " (MEOT, p. 48), c'est-à-dire une nouveauté qui n'est qu'apparente et qui, pour cette raison, n'est pas moins inessentielle à l'objet que son caractère utilitaire. De façon générale, les résultats de décisions pratiques, économiques, sociales, etc. sur le développement des techniques sont inessentiels du point de vue technologique, c'est-à-dire considérés relativement au progrès stricto sensu technique.

${ }^{8} \mathrm{La}$ trace, contrairement à l'observation psychologique, est ce qui permet d'étudier en propre l'invention ainsi que l'une des caractéristiques qui la distingue de la créativité (Simondon, 2018, p. 207).

${ }^{9}$ L'originalité épistémologique de la technologie réside dans le fait que son objet, la compatibilité en question, "n'était pas contenue dans chacun des principes scientifiques séparés qui ont servi à construire l'objet [technique] » (MEOT, p. 58).
} 
l'étude des objets techniques menée dans une perspective technologique c'est-à-dire, selon les mots de Jean-Yves Chateau, qui ne soit pas " une analytique de la puissance, de l'automatisme et de la mise en réseau, du besoin, de l'utilité, de l'usage et du métier ou encore du travail, de la production économique et des structures de production » (Chateau, 1990, p. 106) - rend possible une rationalisation du progrès technique. Cette rationalisation, comme nous allons le voir, requiert une conscience aiguë du rôle que joue l'information au sein des réalités techniques, et en particulier au sein des ensembles.

Les objets techniques, en plus d'informer leur opérateur et de s'informer eux-mêmes par leurs canaux de feedback et par leurs dispositifs d'auto-régulation, s'informent les uns les autres et constituent ainsi des réseaux ou ensembles techniques, qui opèrent à une échelle plus grande que celle de l'individu humain. De cette façon, les ensembles conditionnent l'existence de l'humain ; ils ne se laissent donc pas objectiver aussi facilement que les objets individuels qui les composent. En particulier, leur saisie nécessite cette conscience de l'information par laquelle ils " mettent en situation ", comme dit Simondon (MEOT, p. 311). Sans conscience technologique de cette information, l'humain est affecté par les ensembles techniques comme par des "puissances inconnues et lointaines qui le dirigent sans qu'il les connaisse et puisse réagir contre elles " (MEOT, p. 143), c'est-à-dire de façon négative et aliénante.

Or, à mesure que la technologie étudie la technicité des objets en tant que compatibilité empiriquement découverte entre la pensée et le monde, se forme une culture technique, c'est-à-dire une intuition collective de la technicité des ensembles, en tant que modulateurs de la relation entre l'humain et le monde (MEOT, p. 208). Concrètement, cette culture technique se caractérise par une conscience radicale et partagée de la nature asymptotique des lignées techniques, c'est-à-dire une conscience du fait que l'objet technique "n'est jamais complètement concret » (MEOT, p. 42), que son invention peut toujours être reprise et continuée. L'idée de désaliénation technologique revêt alors une signification précise : celle de l'« invention perpétuée " (MEOT, p. 13). Inventer de façon perpétuée, c'est ne pas finaliser l'existence de la réalité technique ; c'est considérer les techniques comme de l'information ; c'est actualiser un monde dans lequel l'utilisateur est indiscernable de l'inventeur ; c'est contribuer à une culture où la disposition à l'invention est démocratisée. En ceci, les organes de plombages et les contrats de garantie (MEOT, p. 340), par exemple, contredisent la culture technique.

On peut éclairer davantage le sens du réquisit simondonien de conscience de l'information en le comparant à la place de la technique dans la théorie économique de la décroissance de Nicholas Georgescu-Roegen ${ }^{10}$. Ce dernier distingue deux types de progrès techniques : d'un côté, les innovations dites "d'économie nette de basse entropie " correspondent à une combustion plus complète ou encore à une

\footnotetext{
${ }^{10}$ La théorie de Georgescu-Roegen est épistémologiquement fragile, surtout parce que la distinction entre basse et haute entropie, entre " énergie libre » et « énergie liée », repose essentiellement sur la notion d'utilisabilité humaine (NGR, p. 203). Grégoire Wallenborn et Pierre Gillis, notamment, ont montré et discuté les limites méthodologiques de cette tentative anthropocentrique de ratifier l'impossibilité d'un recyclage absolu (Wallenborn et Gillis, 2007).
} 
diminution des frottements; de l'autre, les innovations dites « de substitution» « ne font que remplacer de l'énergie humaine par de l'énergie physicochimique " (NGR, p. 115-116) $)^{11}$. Les deux types de progrès, on le voit, ont en commun la mise en œuvre d'un critère exclusivement thermodynamique. De même, la recherche d'une étude des conditions de l'invention dans la doctrine de Georgescu-Roegen ramène systématiquement à une problématique construite en termes de matière et d'énergie, formulée par exemple à partir de la question : "Comment est-il possible que l'homme produise quelque chose de matériel étant donné qu'il ne peut produire ni matière ni énergie ? » (NGR, p. 66). Georgescu-Roegen ignore ainsi la problématique de la technique comme trace d'une invention. La "basse entropie " ne correspond chez lui qu'à l'état utilisable par l'humain de la "matière-énergie " (NGR, p. 100) ; elle n'est jamais considérée comme de l'information. La technologie de Georgescu-Roegen n'évalue donc de la technique que son rendement énergétique. L'auteur avance par exemple que le progrès technique n'est borné que par le « coefficient théorique de rendement " (NGR, p. 113). Ailleurs, il soutient que, dans l'histoire des techniques, seules la maitrise du feu et la machine à vapeur peuvent être admises au rang des « innovations cruciales » (NGR, p. 210), la maîtrise du feu n'étant appréciée qu'en tant que conversion d'énergie chimique en énergie calorifique et la machine à vapeur qu'en tant que conversion d'énergie calorifique en énergie motrice. Or, selon Simondon,

Toute philosophie des techniques qui part de la réalité des ensembles utilisant les individus techniques sans les mettre en relation d'information reste une philosophie de la puissance humaine à travers les techniques, non une philosophie des techniques (MEOT, p. 176).

Autrement dit, étudier les techniques à partir de la distinction entre énergie utilisable limitée et illimitée, c'est restreindre l'ensemble des techniques à celui des "individus utilisés sans être mis en relation d'information ", c'est se limiter à faire une énergétique ignorante de cette information qu'est la technicité ${ }^{12}$, c'est confondre une axiologie des techniques avec une évaluation des rendements. L'étude génétique de Simondon, à cet égard, explique que concevoir les réalités techniques sans l'information revient à les concevoir comme au XIX ${ }^{\mathrm{e}}$ siècle (MEOT, p. 179). Or, ce siècle était celui des moteurs dont les régulateurs, quand ils existaient, n'étaient encore que de primitifs accessoires. Au XIX ${ }^{\mathrm{e}}$ siècle, les contre-réactions étaient brutalement appliquées sur les organes résistants des machines, la vitesse et l'efficacité dans les régulations étaient très limitées et avaient pour corrélat des opérations extrêmement dépendantes d'un contrôle humain et donc un faible degré d'individualité technique. Au XIX ${ }^{\mathrm{e}}$ siècle, la " voie effective " et celle " de réaction négative " étaient grossièrement confondues ; bref : l'évaluation exclusivement énergétique des techniques se fonde sur une culture du passé, incapable de

\footnotetext{
${ }^{11}$ Nous ne nous arrêtons pas sur les « innovations de la gamme des produits ", " qui créent de nouveaux biens de consommation ", car, explique l'auteur, ce troisième type de progrès peut généralement être rapporté au second.

${ }^{12}$ Il ne faut pas confondre la technique en tant qu'information, c'est-à-dire l'existence de traces d'inventions ou la possibilité de cristalliser des gestes humains, avec les techniques d'information, c'est-à-dire, les réseaux, instruments et outils de communication permettant de convoyer des signaux entre des personnes.
} 
répondre à l'aliénation technologique issue des grands ensembles fondés sur l'information. L'étude simondonienne des différentes notions de progrès technique au fil de l'histoire suggère d'ailleurs l'existence d'une filiation entre la technologie énergétique et le technocratisme ${ }^{13}$ : selon Simondon, le technocratisme aurait justement pour condition technologique d'existence cette "indistinction entre le canal énergétique et le canal d'information » (MEOT, p. 179) qui caractérise l'époque industrielle et productiviste des moteurs. Si Simondon a raison, il parait alors vain de vouloir fonder une critique du technocratisme sur une connaissance des techniques ne reposant elle-même que sur la thermodynamique. Autrement dit, la " [sortie] du gigantisme et de l'hétéronomie auxquels nous ont condamnés l'industrialisation et la technoscience prométhéenne " (Latouche, 2019, p. 89), que la décroissance appelle de ses vœux, requiert notamment une prise de conscience technologique de la nature et des fonctions de la technicité en tant qu'information. Cette prise de conscience exige, comme l'a montré Vincent Bontems, une distinction radicale entre « recherche de progrès " et "quête de puissance " (Bontems, 2016, p. 55). Elle ne contredit pas toutefois le réquisit d'un " accès équitable aux ressources énergétiques ", réquisit que la pensée simondonienne partage tant avec les écologistes de jadis (Simondon, 2014, p. 277) qu'avec les objecteurs de croissance d'aujourd'hui.

Les éléments qui précèdent nous permettent maintenant de répondre à la question posée précédemment au sujet de la relation entre la conception simondonienne du progrès technique et celle inhérente au Grand Récit productiviste critiqué par les objecteurs de croissance. Selon Simondon, personne ne peut rectifier le progrès technique par des décrets ni ne peut le réformer par des prescriptions ou des décisions. Le progrès stricto sensu technique est une concrétisation et une naturalisation; il n'est régi, on l'a vu, que par les nécessités internes aux essences techniques et ne peut conséquemment ni devenir une "marche dans un sens fixé à l'avance " (MEOT, p. 69) ni correspondre à la détermination et au maintien de seuils quantitatifs, à l'instar de celui proposé par Illich dans Énergie et équité (Bontems, 2016, p. 49). Toutefois, le technologue et le philosophe des techniques peuvent contribuer à l'insertion de l'intuition de la technicité dans la culture et participer ainsi à l'instauration d'un processus où l'invention est toujours davantage possible, c'est-à-dire toujours moins interrompue, toujours moins aliénée et, allant, toujours plus démocratisée. " La technique est insuffisante pour porter une éthique mais, avec l'aide de la technologie approfondie, elle apporte cependant une auto-normativité qui est de l'ordre du gai savoir " (Simondon, 2014, p. 351). Chez Simondon, c'est bien la technologie, la connaissance des techniques, qui débouche sur l'esquisse d'une éthique, notamment par une critique de la destruction engendrée par certaines techniques militaires ou polluantes, par l'éloge de la décentralisation des réseaux de ressources, préconisée déjà par l'écologie de son temps, ou encore par l'étude du phénomène d'obsolescence

\footnotetext{
${ }^{13}$ Voir MEOT, p. 178. À la page précédente, on lit : «il ne suffit pas que les techniques se développent pour que le technocratisme naisse. Le technocratisme représente la volonté d'accéder au pouvoir qui se fait jour dans un groupe d'hommes possédant le savoir et non le pouvoir "; et un peu plus loin : « Les technocrates, en France, sont essentiellement des polytechniciens, c'est-à-dire des hommes qui, par rapport aux techniques, sont dans la situation d'utilisateurs intelligents et d'organisateurs plutôt que de véritables techniciens. Ces mathématiciens pensent par ensembles, non par unités individualisées de fonctionnement ; c'est l'entreprise plus que la machine qui retient leur attention » (MEOT, p. 177).
} 
et par la défense corrélative de différentes sortes de "dialectiques de récupération ", c'est-à-dire de recyclages, autant de problèmes indiqués à juste titre et très efficacement par la pensée de la décroissance. Le progrès technique, tel que le conçoit Simondon, ne saurait donc être rapporté au Grand Récit du progrès que critiquent les objecteurs de croissance : l'idée d'un progrès productiviste vise et classe les objets techniques à partir de la croissance ou du rendement qu'ils permettent, en vue de répondre à une exigence externe ; l'idée de progrès stricto sensu technique, par contre, vise et classe des essences techniques en tant que principes de concrétisation à partir d'une étude de la technicité des objets considérée comme de l'information et, d'emblée, inclut systématiquement le milieu. Il revient toutefois à la pensée de la décroissance de rappeler à juste titre que le stock d'énergie immédiatement utilisable dans ce milieu est limité, contrairement aux flux solaires virtuellement illimités, corroborant l'idée simondonienne qu'une pensée ne peut être véritablement technologique que si elle opère à une échelle cosmologique. Par là, la convivialité au même titre que la culture technique mènent bien à une éthique des techniques, et la technicité en tant qu'information peut être entendue à la fois comme formation du monde et comme acculturation de ses habitants.

\section{Conclusion}

Le projet de culture technique de Simondon et celui de décroissance possèdent aussi bien des points de convergence que de divergence. Évoquons de façon très synthétique, pour conclure, les points particulièrement intéressants dans la perspective d'un dialogue constructif.

Tout d'abord, et de façon très générale, Simondon et les objecteurs de croissance accordent une importance méthodologique structurante aux relations qui unissent les individus et les milieux ; en ceci, leurs approches peuvent être dites écologiques au même titre. Toutefois, la conception relationnelle de l'invention, telle que la développe Simondon, inscrit la problématique écologique, en tant que relation entre l'humain et le monde aussi bien qu'entre les techniques et leurs milieux, au cœur du processus génétique d'émergence des réalités techniques. Elle offre ainsi une marge de manœuvre réflexive et effective, capable de moduler les techniques antérieurement à leurs effets et à leurs usages possibles, tout en imposant de coupler l'évaluation technologique des techniques à la compréhension aiguë de leurs milieux de fonctionnement. En ceci, la pensée simondonienne nous paraît pouvoir contribuer de façon constructive aux dimensions technologiques du projet de décroissance.

Ensuite, Simondon, au même titre que les objecteurs de croissance, émet une vive critique du productivisme et de l'idéologie de la puissance. Toutefois, ces critiques opèrent sur deux modes différents : les objecteurs de croissance critiquent le productivisme dans une perspective essentiellement modulée par l'économie et la sociologie. Simondon, quant à lui, émet une critique épistémologique des technologies fondées uniquement sur la thermodynamique, sous la forme d'une étude de leurs limites. Il explique comment la réduction des techniques aux rendements qu'elles permettent manque le rôle et le sens de la technicité en tant qu'information ; il montre en quoi cette information est pourtant nécessaire à la compréhension de l'aliénation inhérente aux grands ensembles du XX $\mathrm{XX}^{\mathrm{e}}$, et désormais du $\mathrm{XXI}^{\mathrm{e}}$ siècle. Cette 
différence méthodologique permet à la critique simondonienne de saisir ce que l'idéologie productiviste inflige aux techniques pour les asservir et, par conséquent, les aliéner. Par exemple, Simondon peut montrer que - mais aussi et surtout comment - le productivisme engendre une artificialisation et une abstraction auxquelles correspond la faiblesse technologique qui est à l'origine d'une partie considérable du coût économique et écologique problématique. À cet égard, la forme de l'analyse de l'artificialité des plantes produites en serre que propose Simondon (MEOT, p. 57) pourrait être étendue à l'ensemble des techniques dites " productivistes"; de telles analyses permettraient alors de critiquer l'aspect indésirable de ces techniques à partir de leur grande dépendance à des conditions ou des médiations humaines, sans sortir toutefois de la technologie. Elles pourraient ainsi compléter, de façon avantageuse, les analyses des usages et des effets que proposent les objecteurs de croissance, notamment via la question de la distinction entre « high tech » et " low tech».

Finalement, Simondon et les objecteurs de croissance se retrouvent ensemble face au constat de la nécessité d'un changement profond dans la relation qui unit les humains aux techniques. L'exigence de produire des techniques assemblables, réutilisables et réparables par tous, issue d'Illich (Illich, 1971, p. 110), converge tout à fait avec le projet de culture technique. Rappelons à cet égard que Simondon a reconnu comme pertinente l'exigence de convivialité formulée par Illich et qu'il a de même soutenu sa critique de l'introduction forcée de techniques dans des populations qui n'en voulaient pas (Simondon, 2014, pp. 345 et 453). La culture technique contribue au dialogue en éclairant la bilatéralité qui caractérise la relation entre l'aliénation technologique de l'humain et celle des techniques. Autrement dit, l'apport de Simondon est la thèse selon laquelle si l'humain subit négativement les ensembles, c'est essentiellement parce que les techniques sont asservies à des puissances non-techniques. La notion d'aliénation des techniques corrobore alors les critiques de l'innovation adressées par les objecteurs de croissance aux productivistes. En outre, l'idée $d^{\prime}$ " invention perpétuée » engage une considération du principe de participation aux techniques dont la radicalité pourrait de même s'articuler aux visées des objecteurs de croissance : selon Simondon, par exemple, la disposition d'un organe de réglage sur une machine ne suffit pas encore à la considérer tout à fait comme une invention perpétuée ${ }^{14}$. Cette radicalité transforme l'abord technologique des techniques en exigence de temps et d'espace plus importants ${ }^{15}$ pour penser et panser les genèses de la culture : le requisit technologique devient alors un requisit symbolique.

\section{Références bibliographiques}

Ariès, P. (2005). Décroissance ou barbarie. Golias.

Aspe, B. (2013). Simondon, politique du transindividuel. Dittmar.

Barthélémy, J.-H. (2005). Penser la connaissance et la technique après Simondon. L'Harmattan. Bayon, D., Flipo, F. et Schneider, F. (2012). La décroissance. La Découverte.

\footnotetext{
${ }^{14}$ Le réglage, écrit Simondon, « est une invention perpétuée, quoique limitée » (MEOT, p. 338).

${ }^{15}$ Bontems montre bien en quoi la conception simondonienne du progrès correspond nécessairement à un ralentissement (Bontems, 2016, p. 54).
} 
Beaune, J.-C. (1990). La philosophie des techniques de Gilbert Simondon. Perspectives sur l'individu et l'invention. Cahiers philosophiques, 43, 47-72.

Beaubois, V. (2015). Un schématisme pratique de l'imagination. Appareil, 16. https://doi.org/10.4000/ appareil.2247

Bihouix, Ph. (2014). L'Âge des low tech. Vers une civilisation techniquement soutenable. Seuil.

Bontems, V. (2016). L'énergétique de Simondon : progrès versus puissance. Dans V. Bontems (dir.), Gilbert Simondon ou l'invention du futur (p. 45-55). Klincksieck.

Carrozzini, G. (2009). Gilbert Simondon et Jacques Lafitte : les deux discours de la "culture technique ». Dans J.-H. Barthélémy (dir), Cahiers Simondon 1 (p. 25-45). L’Harmattan.

Chabot, P. (2003). La philosophie des techniques de Simondon. Dans P. Chabot et G. Hottois (dir.), Les philosophes et la technique (p. 231-242). Librairie philosophique J. Vrin.

Chateau, J.-Y. (1990). Technologie et ontologie dans la philosophie de Gilbert Simondon. Cahiers philosophiques, 43, 97-138.

Chateau, J.-Y. (1994). Technophobie et optimisme technologique modernes et contemporains. Dans G. Châtelet (dir.), Gilbert Simondon. Une pensée de l'individuation et de la technique (p. 115-172). Albin Michel.

Cheynet, V. (2005). Cap sur la décroissance. Manière de voir, 83. https://www.monde-diplomatique.fr/mav/ 83/CHEYNET/56323

Dupin, É. (2009, août). La décroissance, une idée qui chemine sous la récession. Monde diplomatique, $56^{\mathrm{e}}$ année, (665), 20-21.

Flipo, F. (2012). La décroissance, une pensée antimoderne ? Chimères : revue des schizoanalyses, 76, 69-79.

Flipo, F. (s.d.). Décroissance. Dans Encyclopeedia Universalis. https://www.universalis.fr/encyclopedie/ decroissance/ (consulté en 2020).

Georgescu-Roegen, N. (2008). La décroissance. Entropie. Écologie. Économie (traduit par J. Grinevald et I. Rens). Sang de la terre.

Grimaud, E., Tastevin, Y. Ph. et Vidal, D. (dir.). (2017). Low Tech ? Wild Tech ! [Numéro spécial] Techniques et culture, 67.

Guchet, X. (2010). Pour un humanisme technologique. Culture, technique et société dans la philosophie de Gilbert Simondon. Presses universitaires de France.

Guibert, B. (2004). Décoloniser notre imaginaire de croissance ? Ça urge! Mouvements, 33-34, 241-244.

Harribey, J.-M. (s.d.). Critique de la décroissance. Dans Encyclopeedia Universalis. https://www.universalis. fr/encyclopedie/decroissance-critique-de-la/ (consulté en 2020).

Hottois, G. (1993). Simondon et la philosophie de la « culture technique ». De Boeck-Wesmael.

Illich, I. (1971). Une société sans école (traduit par G. Durand). Seuil.

Illich, I. (1973). La convivialité. Seuil.

Illich, I. (2003). Euvres complètes (vol. 1). Fayard.

Labussière, O. (2019). La " part récupérable ». Entre les " mailles " du programme bioéconomique. Multitudes, 77, 61-69.

Latouche, S. (2003). À bas le développement durable ! Vive la décroissance conviviale! Dans M. Bernard, V. Cheynet et B. Clémentin (dir.), Objectif décroissance. Vers une société harmonieuse (p. 19-27). Silence.

Latouche, S. (2019). La décroissance. Que-sais-je ?/Humensis.

Lepesant, M. (2015). Cinq thèses sur la décroissance. Nouveaux Cahiers du socialisme, 14, 41-52.

Marion, L. (2015). La décroissance est-elle technophobe ? Nouveaux Cahiers du socialisme, 14, 86-92.

di Méo, C. (2006). La face cachée de la décroissance. L’Harmattan.

Paquot, Th. (2012). Introduction à Ivan Illich. La Découverte.

Parrochia, D. et Tirloni, V. (dir.). (2012). Formes, systèmes et milieux techniques, après Simondon. Jacques André.

Petit, V. (2016). De l'esthétique industrielle à l'écologie industrielle. Dans V. Bontems (dir.), Gilbert Simondon ou l'invention du futur (p. 125-129). Klincksieck.

Roux, J. (2002). Saisir l'être en son milieu. Voyage en allagmatique simondonienne. Dans P. Chabot (dir.), Simondon (p. 121-135). Librairie philosophique J. Vrin.

Simondon, G. (2005). L'invention dans les techniques. Seuil.

Simondon, G. (2012). Du mode d'existence des objets techniques. Aubier.

Simondon, G. (2014). Sur la technique. Presses universitaires de France.

Simondon, G. (2015). Communication et information. Presses universitaires de France. 
Simondon, G. (2017). L'individuation à la lumière des notions de forme et d'information. Millon.

Simondon, G. (2018). La résolution des problèmes. Presses universitaires de France.

Wallenborn, G. et Gillis, P. (2007). L'économie et la thermodynamique : analyse critique des thèses de Georgescu-Roegen. Cahiers Marxistes, 235, 139-155.

Comment citer cet article ? Alioui, J. (2021). Penser la décroissance avec Gilbert Simondon. Dialogue 60 (3), 483-498. https://doi.org/10.1017/S0012217321000184 